\title{
aA-Crystallin modulates neuroinflammation in the retina via stress-specific inflammatory pathways
}

\author{
Madhu Nath \\ Yang Shan \\ University of Michigan \\ Angela M Myers \\ University of Michigan \\ Patrice E Fort ( $\square$ patricef@umich.edu ) \\ https://orcid.org/0000-0003-3956-1908
}

University of Michigan https://orcid.org/0000-0002-2738-3799

Research

Keywords: aA-crystallin, Müller glial cells, NF-kB, Metabolic stress, Inflammatory markers

Posted Date: April 1st, 2021

DOl: https://doi.org/10.21203/rs.3.rs-345197/v1

License: (c) (i) This work is licensed under a Creative Commons Attribution 4.0 International License. Read Full License 


\section{Abstract \\ Purpose}

We have previously demonstrated that aA-crystallin, a molecular chaperone, plays an important intrinsic neuroprotective role during diabetes, by its phosphorylation on residue 148 . We also reported that aAcrystallin is highly expressed by glial cells. There is a growing interest in the potential causative role of low-grade inflammation in diabetic retinopathy pathophysiology and retinal Müller glial cells (MGCs) participation in the inflammatory response. MGCs indeed play a central role in retinal homeostasis via secreting various cytokines and other mediators. Hence, this study was carried out to delineate and understand the regulatory function of aA-crystallin in the inflammatory response associated with metabolic stresses.

\section{Methods}

Primary MGCs were isolated from Ko-aA-crystallin mice. These primary cells were then transfected with plasmids encoding either wild-type (WT), phosphomimetic (T148D), or non-phosphorylatable mutants (T148A) of aA-crystallin. The cells were exposed to multiple metabolic stress including serum starvation (SS) or high glucose with TNF-alpha $(\mathrm{HG}+\mathrm{T})$ before being further evaluated for the expression of inflammatory markers by qPCR. The total protein expression along with subcellular localization of NF-kB \& NLRP3 component was assessed by western blot.

\section{Results}

Elevated levels of IL-6, IL-1 $\beta$, MCP-1, and IL-18 in SS were significantly diminished in MGCs overexpressing WT and further in T148D as compared to EV. The HG + T-induced increase in these inflammatory markers was also dampened by WT and even more significantly by T148D overexpression, whereas T148A was ineffective in either stress. Further analysis revealed that overexpression of WT or the T148D, also lead to a significant reduction of Nlrp3, Asc, and caspase-1 transcript expression in serum-deprived MGCs and nearly abolished the NF-kB induction in HG + T diabetes-like stress. This mechanistic effect was further evaluated at the protein level and confirmed the stress-dependent regulation of NLRP3 and NF-kB by aA-crystallin.

\section{Conclusion}

The data gathered in this study demonstrate the central regulatory role of aA-crystallin and its modulation by phosphorylation on T148 in retinal MGCs. For the first time, this study demonstrates that aA-crystallin can dampen the stress-induced expression of pro-inflammatory cytokines through modulation of multiple 
key inflammatory pathways, therefore, suggesting its potential as a therapeutic target for modulation of chronic neuroinflammation.

\section{Introduction}

Unique longitudinal positioning of the Müller glia Cells (MGCs) in the retina, enables them to interact with every cellular structure of the retina and hence, makes them a key player in maintaining retinal homeostasis. MGCs are the major glial cell of the retina and as such are involved in multiple processes such as neurotransmitter recycling, preventing glutamate toxicity (1), maintaining the retinoid cycle (2), and regulating nutrient supply $(3,4)$ in a healthy retina. Also, MGCs along with pericytes and endothelial cells, constitute the blood-retinal barrier and prevent retinal cells from getting exposed to potentially harmful molecules and pathogens (5). Therefore, any acute or chronic injury to the retina would primarily influence the proper functioning of MGCs and can affect the entire retina's well-being.

During prolonged or extensive retinal injury such as diabetic retinopathy (DR), MGCs stress response is primary protective through secretion of growth factors and regulation of other pro-survival effects, but can eventually become detrimental including through its participation in the inflammatory response with the secretion of various cytokines, and other mediators in the extracellular space and the vitreous cavity. Studies have reported that diabetes-induced gliosis eventually leads to the production and release by MGCs of inflammatory molecules such as interleukin-1 $\beta$ (IL-1 $\beta$ ), interleukin-6 (IL-6), tumor necrosis factor$a(T N F-a)$, and chemokine ligand-2 (CCL2) $(6,7)$. The discovery of these same elevated inflammatory molecules and cytokines in the vitreous of diabetic patients at various stages of DR strongly supports that MGCs play a key role in the synthesis of these cytokines, and more generally, the neuroinflammatory response associated with DR (8-12).

Lately, there has been a growing interest in the role of crystallin proteins in glia and especially their implication in neuroinflammation. a-Crystallins have particularly received increasing attention as they were shown to be highly expressed in glial cells in multiple neurodegenerative diseases such as multiple sclerosis (13), Alzheimer's disease (14), and diabetic retinopathy $(15,16)$. a-Crystallins are multifunctional proteins that possess remarkable chaperone and anti-apoptotic properties (15-18). These proteins are involved in various healthy and stress-associated cellular mechanisms including regulation of protein aggregation and oxidation, through which they can play a central role in inflammation and apoptosis, including those involved in neurodegenerative disorders. Studies have reported that pre-treatment of aAcrystallin significantly diminishes the silver nitrate stimulated acute systemic inflammation in mice models. The pre-treatment of aA-crystallin also effectively reduced the glial fibrillary acidic protein (GFAP), nuclear factor kappa B (NFkB), and dopamine, norepinephrine catabolism in the mice neocortex $(19,20)$.

In the retina, an aA-crystallin presence was reported in the frog (21) as well as mice and rat MGCs (22). Our group previously reported that aA-crystallin was highly expressed in the inner retina of diabetic rats and was primarily located in the ganglion cells and MGCs $(15,16)$. a-Crystallins undergo numerous post- 
translation modifications (PTMs), which can affect their chaperone activity $(9,23-26)$. Phosphorylation of aB-crystallin at Serine residues 19 and 45 was shown to be required for multiple functions including its protective role in myocytes (27) or it's regulation of lens epithelial cell migration (28). More recently, phosphorylation of serine residues at positions 19,45 , and 59 of aB-crystallin was reported to differentially regulate its chaperone activity for transmembrane proteins (25). Studies of rodent models of diabetes reported increased phosphorylation of aB-crystallin on serine residues 19, 45, and 59 in the retina $(15,29,30)$. Conversely, we recently reported that phosphorylation on the serine/threonine 148 residue of aA-crystallin is high in the retina under normal conditions, while it is dramatically reduced in diabetic donors, especially those with retinopathy (16). Further exploration revealed that serine/threonine 148 phosphorylation essentially controls the protective role of aA-crystallin, highlighting the physiological importance of its down-regulation under metabolic stress and diabetic conditions (16). We have demonstrated that aA-crystallin promotes retinal ganglion cell survival under metabolic stress through dampening of the associated endoplasmic reticulum stress. While highly expressed by MGCs, aAcrystallin had only a limited effect on ER stress in these cells (16), suggesting a different function of aAcrystallin in MGCs.

aA administration in the experimental autoimmune uveitis mice model caused the marked reduction in Th1 cytokines (TNF-a, IL-12, and IFN- $\gamma$ ), both in the retina and in the spleen (31). Because of the previously demonstrated anti-inflammatory role of aA-crystallin in systemic inflammation, we hypothesized that aA-crystallin plays a central role in the regulation of the inflammatory response of retinal MGCs during diabetes and metabolic disorders. Therefore, the present study investigated the effect of aA-crystallin on the neuroinflammatory response in retinal MGCs under metabolic stress.

\section{Methods And Materials}

\section{Cell culture:}

Rat retinal Müller cells (rMC-1) were purchased from Applied Biological Material Inc. The rMC-1 cell line is derived from the stable transformation of SV40 antigen into primary rat retinal Müller cells shown to express GFAP and cellular retinaldehyde-binding protein (CRALBP), two markers for Müller cells in the adult retina (32). While originally maintained in $25 \mathrm{mM}$ glucose, cells were progressively acclimated to be cultured and maintained in DMEM containing $5 \mathrm{mM}$ glucose supplemented with $10 \%$ FBS.

Primary Müller glial cells (MGCs) were obtained from the aA-crystallin knockout mice originally generously provided by Dr. Wawrousek from the National Eye Institute (NEI). Cells were isolated using a protocol adapted from Hicks and Courtois (33) and characterized as described previously (34). Briefly, primary MGCs were isolated from the retinal tissue of P10-14 aA-crystallin knockout mice pups and maintained in DMEM (5mM Glucose) $+10 \%$ FBS + 1\% Penicillin/Streptomycin. The primary Müller cells culture was analyzed for the expression of Müller cells specific gene expression from passage 2 to 6 .

\section{Transfection \& Experimental Protocol:}


Cells were transfected using the Neon Transfection System (Invitrogen) following the manufacturer's instructions. Briefly, cells were trypsinized and washed in PBS before being resuspended in suspension buffer and electroporated with targeted plasmids. Cells were then plated in 6-well plates for gene expression studies. Following day post-transfection, cells were plated in DMEM with $5 \mathrm{mM}$ or $25 \mathrm{mM}$ glucose for 24 hours. The cells were then incubated in either serum-free DMEM, $25 \mathrm{mM}$ glucose, or $25 \mathrm{mM}$ glucose with 100ng/ml TNFa (R\&D Systems, Catalog \# 210-TA) for four hours before analysis, whereas $5 \mathrm{mM}$ DMEM served as the experimental control. Cells were then harvested for the gene expression studies.

\section{Subcellular fractionation:}

Cells were subjected to the REAP (Rapid, Efficient, And Practical) method for subcellular nuclear and cytosolic fractionation as described by Suzuki et al (2010) (35). Briefly, the cell pellets were resuspended in $900 \mu \mathrm{L}$ of ice-cold $0.1 \%$ NP40 (Calbiochem, CA, USA) in PBS and triturated 5 times using a p1000 micropipette. $300 \mu \mathrm{L}$ of the lysate was removed as "whole cell lysate". The remaining $(600 \mu \mathrm{L})$ material was centrifuged for $10 \mathrm{sec}$ in $1.5 \mathrm{ml}$ micro-centrifuge tubes and $300 \mu \mathrm{l}$ of the supernatant was removed as the "cytosolic fraction". After the remaining supernatant was removed, the pellet was resuspended in 1 $\mathrm{ml}$ of ice-cold $0.1 \%$ NP40 in PBS and centrifuged as above for $10 \mathrm{sec}$ and the supernatant was discarded. The pellet $(\sim 20 \mu \mathrm{L})$ was resuspended with $180 \mu \mathrm{L}$ of ice-cold $0.1 \%$ NP40 in PBS and designated as "nuclear fraction".

\section{Immunoblot:}

Cells were homogenized by sonication in the previously described RIPA buffer (16). Protein concentrations were measured with the Pierce BCA reagent, and all samples were adjusted for equal protein concentration. Whole lysates and subcellular fractions were immunoblotted using NuPage gels $4 \%-12 \%$ and MES buffer following the manufacturer's instructions (Thermo Fisher Scientific, USA). Gels were run in MES buffer (Thermo Fisher Scientific, USA) as per the manufacturer's instructions. Western blot transfer was carried out on Nitrocellulose membranes using the Mini Trans-Blot cell (Catalogue \# 1703930, Biorad, USA) at $160 \mathrm{~V}$ for 1 hour at $4^{\circ} \mathrm{C}$. Cell lysates were screened for nuclear factor $\mathrm{KB}$ (D14E12, Cell signaling technology, USA), phosphorylated nuclear factor KB (Ser536) $(94 \mathrm{H} 1$, Cell signaling technology, USA), NOD-, LRR- and pyrin domain-containing protein 3 (D25PE, Cell signaling technology, USA), GAPDH (D16H11, Cell signaling technology, USA), Histone H3 (D1H2, Cell signaling technology, USA), aA-crystallin (sc-28306, Santacruz Biotechnology, USA) expression and $\beta$-actin (MAB1501, Millipore, USA) as a loading control.

\section{qPCR:}

The pooled cell lysate samples $(n=3)$ were immediately placed in TRIzol (Thermo Scientific, USA) and subsequently, RNA purification was done using the phenol-choloroform method (36). Isolated RNA samples were quantified by using NanoDrop 1000 Spectrophotometer (Thermo Fisher Scientific, USA). RNA samples of sufficient purity (A260/A280 ratio of 1.9-2.1) were used for the synthesis of cDNA 
(Thermo Fisher Scientific, USA). Contaminating DNA was degraded by treating each sample with RQ1 RNase-free Dnase (Promega) according to the instruction's manual. Total RNA isolated from the cell lysates were reverse transcribed into cDNA (Qiagen omnscript RT kit, 205111) as per the manufacturer's instructions.

The synthesized cDNAs amplification was performed using SYBR green master mix (Applied Biosciences A25742) and was used for qPCR analysis (Thermocycler, Biorad, USA). Real-time experiments were performed in triplicate for each gene including the housekeeping gene ( $\beta$-actin). The threshold cycle at which the increase in the signal with the exponential growth of PCR products was detected was obtained for the quantification. The values were normalized by those in the control group. Relative Gene Expression Analysis was done by 2-Delta Delta $\mathrm{C}(\mathrm{t})$ method. The primer sequences for gene tested are listed in Table 1.

\section{Statistics:}

Gene expression experiments data were normalized to the signal of housekeeping gene $\beta$-actin and then further with the control group to obtain the Delta Delta $\mathrm{C}(\mathrm{t})$ The mean \pm SEM and statistically significant differences are reported. Analyses were performed using non-repeated- measures ANOVA, followed by the Student-Newman-Keuls test for multiple comparisons or 2-tailed t-test for a single comparison. A p-value less than 0.05 was considered significant.

\section{Study approval:}

All experiments were conducted following the Association for Research in Vision and Ophthalmology Resolution on the Care and Use of Laboratory Animals and approved by the Institutional Animal Care and Use Committee of the University of Michigan.

\section{Results}

\section{aA-crystallin overexpression dampens the metabolic-stress induced pro-inflammatory transition of Müller Glial cells (MGCs).}

We have previously reported that aA-crystallin is highly expressed by retinal MGCs under diabetic conditions. We also showed that rat retinal Müller cells ( $\mathrm{rMC}-1)$ overexpressing aA-crystallin can protect R28 rat retinal neuron cells from metabolic stress (16). Since, MGCs play a vital role in retinal homeostasis, particularly regarding metabolism, neurotransmission, and inflammation, we further investigated the effect of the aA-crystallin expression on the inflammatory response of MGCs to metabolic stress. We observed that metabolic stress-induced by serum deprivation leads to elevated expression of pro-inflammatory cytokines including interleukin-6 (IL-6), IL-1beta (IL-1 $\beta$ ), and monocyte chemoattractant proteins-1 (MCP-1) in rMC-1 (Figure 1). Interestingly, this serum deprivation-induced increase expression of pro-inflammatory mediators was significantly prevented by WT aA-crystallin 
overexpression as evidenced by $57 \%, 80 \%$, and $82 \%$ reduction in levels of IL-6, IL-1 $\beta$, and MCP-1 respectively, as compared to the empty vector.

Since aA-crystallin is induced in MGCs during diabetes, we also studied the effect of aA-crystallin overexpression on metabolic stress conditions more reminiscent of diabetes, that is high glucose and TNFa. Our data show that exposure of rMC-1 to a "diabetes-like condition" resulted in elevated levels of IL$1 \beta$ and IL- 6 comparable to serum starvation. Of note, a much more dramatic increase in MCP-1 expression was observed (352\%) in "diabetes-like" conditions when compared to serum starvation. Consistent with a key role of aA-crystallin in the regulation of MGCs activation in metabolic stress, WT aA-crystallin overexpression significantly reduced the induction of IL-6 (61\%), IL-1 $\beta$ (77\%), and MCP-1 (63\%) in "diabetes-like" condition as compared to the respective empty vector.

\section{aA-crystallin effect on the metabolic-stress induced activation of Müller Glial cells is T148 phosphorylation-dependent.}

We have previously reported that Thr148 phosphorylation of aA-crystallin was reduced dramatically in human donors with diabetic retinopathy. We further demonstrated that the phosphorylation of aAcrystallin on residue 148 controls its neuroprotective function in R28 rat retinal neuron cells (16). Thus, we next assessed the role of this phosphorylation on the dampening effect of aA-crystallin on the metabolic stress-induced pro-inflammatory response of MGCs. To do so, we overexpressed wild-type aAcrystallin (WT), the phosphomimetic (148D) aA-crystallin, or the non-phosphorylatable (148A) aAcrystallin mutant in rMC-1 cells (Figure 1D). Supporting a key role of this phosphorylation, the phosphomimetic (148D) aA-crystallin mutant had an even greater dampening effect than the WT protein on the expression of these pro-inflammatory cytokines, reducing their serum-deprivation induction by $64 \%, 86 \%$, and $79 \%$ for IL-6, IL-1 $\beta$ and MCP-1 respectively. Conversely, the non-phosphorylatable (148A) aA-crystallin mutant was wildly ineffective at reducing the induction of any of these pro-inflammatory cytokines in serum deprivation stress. Similarly, in a "diabetes-like" condition, the phosphomimetic (148D) aA-crystallin mutant had an even greater dampening effect than that of the WT aA-crystallin as demonstrated by a greater decrease in the induction of IL- 6 (78\%), IL-1 13 (79\%), and MCP-1 (83\%). Conversely again, the levels of induction of IL-6, IL-1 $\beta$, and MCP-1 by "diabetes-like" conditions in presence of the non-phosphorylatable (148A) mutant were comparable to those obtained in absence of aA-crystallin (EV).

\section{Primary Müller cells isolated from aA-crystallin knockout mice}

The data obtained from the rat retinal Müller cell lines ( $\mathrm{rMC}-1$ ) were consistent with a key role of aAcrystallin in Müller glial cells, especially in the modulation of their inflammatory response associated with metabolic stress. To further assess the function of aA-crystallin in a more physiologically relevant system, we continued our study in freshly isolated primary MGCs. Additionally, the primary MGCs were isolated from the retinas of aA-crystallin knockout mice to prevent any potential interference of the endogenously expressed aA-crystallin. The quality and stability of the primary MGCs were controlled by analysis of the expression of specific markers upon isolation. The analysis of expression of gene-specific 
to retinal MGCs, that is $\operatorname{Prdx}-6, G L U L$, and $A b c 8 a$ revealed that these cells maintain their specificities in our culture conditions until passage 6 (Figure 2B-D). The dramatic reduction of expression of the specific markers at passage 6 was associated with a change in morphology as the cells became flat and nonpolarized (Figure 2A). Henceforth, all experiments were performed with primary MGCs before passage 6 .

\section{The metabolic-stress induced pro-inflammatory response of primary MGCs is dampened by aA-crystallin expression}

Similar to what was observed in rMC-1, serum deprivation also induced increased expression of multiple pro-inflammatory cytokines by primary MGCs. Of note, the relative induction of IL-1 $\beta$, IL- 6 , and MCP-1 seem even more dramatic in MGCs $(\approx 10-15$ fold) as compared to rMC-1 cells $(\approx 5-8$ fold), potentially due to the lack of endogenous aA-crystallin. Despite this dramatic increase, overexpression of WT aAcrystallin in primary MGCs nearly abolished the serum-deprivation induction of these pro-inflammatory cytokines as it resulted in $97 \%, 88 \%, 72 \%$, and $89 \%$ reduction in expression of IL-6, IL-1 3 , IL-18, and MCP-1 respectively (Figure 3). Primary MGCs seem to be also very highly reactive to the "diabetes-like" conditions as compared to rMC-1 cells, with even greater induction of the different cytokines analyzed. Under "diabetes-like" conditions, WT aA-crystallin overexpression led to the significant reduction of the induction of IL-6 (55\%), IL-1 $\beta$ (36\%), IL-18 (93\%), and MCP-1 (85\%) by MGCs (Figure 3).

\section{Phosphorylation of aA-crystallin on residue 148 controls the neuroinflammatory cascade in metabolically stressed Primary MGCs.}

Next, we assessed if phosphorylation on residue 148 is necessary for the regulation by aA-crystallin of the metabolic-stress induced neuroinflammatory response of primary MGCs. Consistent with the key role of this phosphorylation, the metabolic-stress-induced inflammatory response of MGCs was significantly dampened by the phosphomimetic (148D) but not the non-phosphorylatable (148A) aA-crystallin mutant. The phosphomimetic (148D) mutant reduced IL-6, IL-1 $\beta$, IL-18, and MCP-1 expression by $87 \%, 86 \%, 64 \%$, and $28 \%$ respectively, while the expression of these inflammatory mediators remained comparable to the empty vector control in MGCs overexpressing the non-phosphorylatable (148A) aA-crystallin mutant. Under "diabetes-like" stress, the phosphomimetic (148D) aA-crystallin mutant was even more effective at reducing the pro-inflammatory mediator's response of MGCs than the WT aA-crystallin protein. While the phosphomimetic (148D) aA-crystallin overexpressing MGCs showed a greater reduction of the "diabeteslike" stress-induced pro-inflammatory mediators evaluated, the non-phosphorylatable (148A) aA-crystallin mutant was completely devoid of dampening effect (Figure 3A-D).

\section{aA-crystallin regulates MGCs inflammatory response through stress-specific inflammatory pathways}

Previous studies have reported that diabetes and metabolic stress can cause the induction of proinflammatory cytokines through activation of nuclear factor kappa B (NF-kB) or the NLRP3 inflammasomes (37-39). In addition to demonstrating the key role of aA-crystallin in regulating the proinflammatory response of MGCs to metabolic stress, our data also demonstrated that it did so despite a cytokine profile varying based on the nature of the stress. This stress-specificity of the response 
prompted us to assess the role of aA-crystallin in the expression and activation of these respective pathways. This analysis showed that serum deprivation led to robust activation of the NLRP3 inflammasome (Figure 4A-C) while diabetes-like stress caused a specific and dramatic induction of NF-kB (Figure 4D). While aA-crystallin overexpression led to some degree of variability in the expression levels of various effectors of the inflammasome under diabetes-like conditions, none of them were significantly different from normal EV conditions. Conversely, serum deprivation had only a very moderate effect on NF-KB expression compared to the effect of diabetes-like stress. Consistent with its impact on proinflammatory cytokines expression, overexpression of WT aA-crystallin or the phosphomimetic mutant, lead to a significant reduction of Nlrp3 (85\%), Asc (61\%), and caspase-1 (68\%) induction in serumdeprived MGCs (Figure 4A-C). Overexpression of phosphomimetic mutant significantly decreased the cytosolic Nlrp3 protein expression by $\approx 2$-fold (Figure 5A-B). Similarly, overexpression of WT aA-crystallin or the phosphomimetic mutant nearly abolished the "diabetes-like" stress induction of NF-kB. Underlining the key role of T148 phosphorylation, and consistent with the effects on cytokine expression, overexpression of the non-phosphorylatable (148A) aA-crystallin mutant was virtually completely ineffective under both stress conditions (Fig 4A-D). At the protein level activation of NF-kB was significantly reduced by the phosphomimetic mutant by inhibiting NF-kB phosphorylation at Ser536 under the serum starvation and "diabetes-like" stress, where the presence of non-phosphorylatable (148A) aA-crystallin mutant had no effect (Figure 5C-F).

\section{Discussion}

The present study demonstrated the critical role of aA-crystallin in the regulation of metabolic stressinduced inflammatory cascade in retinal Müller Glial cells. This study revealed that aA-crystallin overexpression leads to the dampening of the metabolic-stress-induced pro-inflammatory transition of retinal MGCs. It also clearly established that this function is tightly regulated by the phosphorylation of aA-crystallin on residue 148 , phosphorylation required to suppress the neuroinflammatory cascade in metabolically stressed MGCs. Interestingly, our study also demonstrated the pleiotropic nature of this regulation demonstrated by its efficacy in two different models of metabolic stresses involving partially separate inflammatory pathways. Overall, our study suggests that aA-crystallin and its phosphorylation on residue 148 could play a central role in regulating the stress response of retinal MGCs during diabetes and other metabolic disorders.

Our study has demonstrated that retinal MGCs upon exposure to metabolic stress initiate proinflammatory cascades. The finding is consistent with previous reports, describing the activation of retinal MGCs during neurodegenerative diseases such as diabetic retinopathy (40-43). Various studies have reported increased expression of inflammatory markers such as IL-6 (7), IL-1 $\beta$ (44), and MCP-1 (41) inactivated retinal MGCs due to diabetes-induced stress. This cytokine profile is consistent with the one reported in the current study when primary MGCs are exposed to hyperglycemia and high TNFa, subsequently referred to as "diabetes-like" stress conditions. Altogether, recent studies are strongly supportive of the notion that an inflammatory cascade in activated retinal MGCs is involved in the 
pathogenesis of diabetic retinopathy $(5,45)$. Our group has previously demonstrated that aA-crystallin is predominantly expressed in ganglion cells and MGCs in diabetic patients (16).

While aA-crystallin function in retinal MGCs is largely unknown, an active role of aA-crystallin in suppressing systemic inflammation has previously been reported $(19,20)$. Together with the findings of the current study, it supports a regulatory role of aA-crystallin in the inflammatory response of MGCs in the stressed retina. Reactive astrogliosis, while disease and stimulus-dependent, is most often associated with a pro-inflammatory $\mathrm{A} 1$ phenotype rather than the more protective, anti-inflammatory A2 phenotype. Similarly, to inflammation itself, while first necessary and protective, sustained or unresolved reactive gliosis becomes part of the pathophysiology of neurodegeneration, including in diabetic retinopathy. The current study is the first to show the potential role of aA-crystallin in regulating retinal MGCs activation, including preventing or suppressing the transition to the pro-inflammatory A1 reactive phenotype associated with metabolic stress.

Post-translational modifications in general, and phosphorylation in particular, are key mechanisms of regulation of protein function, a phenomenon well-characterized for aB-crystallins. Phosphorylation of aB-crystallin can be induced by MAPKs including extracellular signal-regulated kinase (ERK) and p38 under stress conditions $(46,47)$, which eventually regulates its intracellular distribution, translocation, chaperone activity $(48,49)$ and also enhances its anti-apoptotic potential $(50)$. Although, earlier in-vivo studies had shown aA-crystallin in the lens can be phosphorylated at residue-122 and 148, unlike aBcrystallin, detailed functional studies for aA-crystallin phosphorylation are very limited (51-53). aAcrystallin expression was reported to protect astrocytes against C2-ceramide- and staurosporine-induced cell death (44), and subsequent work suggested that phosphorylation of aA-crystallin at residues 122 and 148 enhanced this protective effect (48). Our group was first to report that Thr148 phosphorylation of aAcrystallin was reduced dramatically in the retina of human donors with diabetic retinopathy (16). While this study also demonstrated that aA-crystallin phosphorylation on this specific residue played a central role in regulating its protective function in neurons, it did not unveil the intracellular role of aA-crystallin and its phosphorylation in MGCs. Using primary MGCs, the current study demonstrated the physiological role of aA-crystallin in regulating the inflammatory response of those cells when exposed to metabolicstress conditions.

Retinal inflammation is a hallmark of various retinal pathologies including diabetic retinopathy, agerelated macular degeneration, uveitis, and retinopathy of prematurity. Despite sharing the common feature of retinal inflammation, these diseases vary regarding the pro-inflammatory cytokine profiles associated and the inflammatory pathways involved. This is consistent with our study, as both metabolic stresses tested showed an overall increase in pro-inflammatory cytokines expression, however with different patterns. Most notably, the more chronic "diabetic-like" stress was strikingly associated with a greater increase in MCP-1. Interestingly, this difference was correlated with the marked induction of Nuclear factor-kappa B (NF-kB). This observation suggests that MGCs could be directly responsible for the previously reported diabetes-induced elevation of NF-kB, which has been suggested to result in the associated increased levels of MCP-1 (37). This is further supported by the observation of local 
expression of MCP-1 and NF-kB in the retina as well as their accumulation in the vitreous of patients suffering from proliferative diabetic retinopathy $(54,55)$.

In the more acute metabolic stress induced by serum deprivation, the significant induction in the IL-1 $\beta$, IL6 , and IL-18 was particularly associated with an increase in NLRP3 inflammasome induction. Retinal MGCs are an essential element of retinal innate immunity (56), especially so in response to acute stress that can result in reactive gliosis, including through the induction of IL-1 $\beta, I L-6, T N F-a$, and IL-18 (57). The results of the current study also suggest that MGCs can play an important role in regulating the expression of pro-inflammatory cytokines such as IL-1 $\beta$, IL-6, TNF- $a$, and IL-18 in the early stages of diabetic retinopathy, which was proposed to be associated with the inflammasome activation $(58,59)$. Recent studies in streptozotocin-induced diabetic rodents showed increased retinal expression of NLRP3, caspase-1, and ASC (44), whereas, dysregulation of NLRP3 inflammasome in the Akimba mice, led to the continuous activated state of microglia resulting in loss of blood-retinal barrier (60). Studies focusing on age-related macular degeneration have also reported NLRP3 inflammasomes dependent cytokine induction of IL-1 $\beta$ and IL-18, both locally and systemically $(38,39)$. Our data suggest that aA-crystallin can also dampen the inflammatory mediator induction in the more chronic and "diabetic-like condition" controlled by NF-kB, a key modulator of the expression of cytokines and inflammatory molecules associated with diabetes complications $(61,62)$. Our data also highlight the role of aA-crystallin in the regulation of inflammasome induction, which plays an important role in the more acute inflammatory response of MGCs to metabolic stress-driven insult occurring in the early stages of DR.

\section{Conclusion}

In conclusion, the data gathered in this study demonstrate the central regulatory role of aA-crystallin in MGCs, as it significantly dampens the sustained expression of pro-inflammatory cytokines and its detrimental effects through modulation of major inflammatory response pathways. Additionally, this study points to a novel mechanism of regulation of this new role of aA-crystallin, its phosphorylation on $\mathrm{T} 148$, and its potential use as a therapeutic target for modulation of chronic neuroinflammation.

\section{Declarations}

\section{Ethics declaration and consent to participate}

All experiments were conducted following the Association for Research in Vision and Ophthalmology Resolution on the Care and Use of Laboratory Animals and approved by the Institutional Animal Care and Use Committee of the University of Michigan. No human participants

\section{Consent for publication}

This publication does not contain any individual person's data, therefore is not applicable.

\section{Availability of data and materials}


All data generated or analysed during this study are included in this published article

\section{Competing interests}

All the authors declare that they do not have any competing interests.

\section{Funding}

This work was supported by the National Institutes of Health (R01EY020895, the Kellogg Eye Center Core Center for Vision Research P30EY007003, the Michigan Diabetes Research Center P30DK020572), and a research grant by the Thomas Beatson Foundation. The funding bodies had no other involvement in any aspect of the study or preparation of the manuscript.

\section{Author's contributions}

MN performed experiments, participated to interpretation, prepared the original figures and wrote the draft of the manuscript. YS prepared the primary cultures and helped with preparation of the figures and edited the manuscript. AMM helped with tissue recovery for primary culture, experimental procedures and preparation of the figures and edited the manuscript. PEF is the guarantor of this work, provided funding, designed experiments, helped with interpretation and preparation of the figures and edited and finalized the manuscript.

\section{Acknowledgments}

The authors thank Dr. Eric Wawrousek for providing the aA-crystallin knockout mice used for the primary cultures.

\section{Abbreviations}

MGCs- Müller glia Cells

IL-1 $\beta$ - Interleukin-1 $\beta$

IL-6- interleukin-6

TNF- $a$-Tumor necrosis factor- $a$

CCL2- Chemokine ligand-2

DR-Diabetic Retinopathy

NFkB- Nuclear factor kappa B

PTMs- Post-translation modifications

CRALBP-Cellular Retinaldehyde-Binding Protein 
REAP-(Rapid, Efficient, And Practical) method

rMC-1- Rat retinal Müller cells

NLRP3- NOD-, LRR- and pyrin domain-containing protein 3

MCP-1- Monocyte chemoattractant proteins-1

WT - Wild-type aA-crystallin

148D-Phosphomimetic aA-crystallin

148A -Non-phosphorylatable aA-crystallin

Asc- Apoptosis-Associated Speck-Like Protein Containing CARD

\section{References}

1. Reichenbach A, Bringmann A. Role of Purines in Muller Glia. J Ocul Pharmacol Ther. 2016;32(8):51833.

2. Xue Y, Shen SQ, Jui J, Rupp AC, Byrne LC, Hattar S, et al. CRALBP supports the mammalian retinal visual cycle and cone vision. J Clin Invest. 2015;125(2):727-38.

3. Hurley JB, Lindsay KJ, Du J. Glucose, lactate, and shuttling of metabolites in vertebrate retinas. J Neurosci Res. 2015;93(7):1079-92.

4. Lindsay KJ, Du J, Sloat SR, Contreras L, Linton JD, Turner SJ, et al. Pyruvate kinase and aspartateglutamate carrier distributions reveal key metabolic links between neurons and glia in the retina. Proc Natl Acad Sci U S A. 2014;111(43):15579-84.

5. Subirada PV, Paz MC, Ridano ME, Lorenc VE, Vaglienti MV, Barcelona PF, et al. A journey into the retina: Muller glia commanding survival and death. Eur J Neurosci. 2018;47(12):1429-43.

6. Yego EC, Vincent JA, Sarthy V, Busik JV, Mohr S. Differential regulation of high glucose-induced glyceraldehyde-3-phosphate dehydrogenase nuclear accumulation in Muller cells by IL-1 beta and IL6. Invest Ophthalmol Vis Sci. 2009;50(4):1920-8.

7. Lei $X$, Zhang J, Shen J, Hu LM, Wu Y, Mou L, et al. EPO attenuates inflammatory cytokines by Muller cells in diabetic retinopathy. Front Biosci (Elite Ed). 2011;3:201-11.

8. Abu el Asrar AM, Maimone D, Morse PH, Gregory S, Reder AT. Cytokines in the vitreous of patients with proliferative diabetic retinopathy. Am J Ophthalmol. 1992;114(6):731-6.

9. Blakytny R, Carver JA, Harding JJ, Kilby GW, Sheil MM. A spectroscopic study of glycated bovine alpha-crystallin: investigation of flexibility of the C-terminal extension, chaperone activity and evidence for diglycation. Biochim Biophys Acta. 1997;1343(2):299-315.

10. Deliyanti D, Alrashdi SF, Tan SM, Meyer C, Ward KW, de Haan JB, et al. Nrf2 Activation Is a Potential Therapeutic Approach to Attenuate Diabetic Retinopathy. Invest Ophthalmol Vis Sci. 2018;59(2):815- 
25.

11. Demircan N, Safran BG, Soylu M, Ozcan AA, Sizmaz S. Determination of vitreous interleukin-1 (IL-1) and tumour necrosis factor (TNF) levels in proliferative diabetic retinopathy. Eye (Lond). 2006;20(12):1366-9.

12. Hernandez C, Segura RM, Fonollosa A, Carrasco E, Francisco G, Simo R. Interleukin-8, monocyte chemoattractant protein-1 and IL-10 in the vitreous fluid of patients with proliferative diabetic retinopathy. Diabet Med. 2005;22(6):719-22.

13. van Noort JM, van Sechel AC, Bajramovic JJ, el Ouagmiri M, Polman CH, Lassmann H, et al. The small heat-shock protein alpha B-crystallin as candidate autoantigen in multiple sclerosis. Nature. 1995;375(6534):798-801.

14. Renkawek K, Stege GJ, Bosman GJ. Dementia, gliosis and expression of the small heat shock proteins hsp27 and alpha B-crystallin in Parkinson's disease. Neuroreport. 1999;10(11):2273-6.

15. Fort PE, Freeman WM, Losiewicz MK, Singh RS, Gardner TW. The retinal proteome in experimental diabetic retinopathy: up-regulation of crystallins and reversal by systemic and periocular insulin. Mol Cell Proteomics. 2009;8(4):767-79.

16. Ruebsam A, Dulle JE, Myers AM, Sakrikar D, Green KM, Khan NW, et al. A specific phosphorylation regulates the protective role of alphaA-crystallin in diabetes. JCI Insight. 2018;3(4).

17. Nagaraj RH, Nahomi RB, Mueller NH, Raghavan CT, Ammar DA, Petrash JM. Therapeutic potential of alpha-crystallin. Biochim Biophys Acta. 2016;1860(1 Pt B):252-7.

18. Nahomi RB, Wang B, Raghavan CT, Voss O, Doseff Al, Santhoshkumar P, et al. Chaperone peptides of alpha-crystallin inhibit epithelial cell apoptosis, protein insolubilization, and opacification in experimental cataracts. J Biol Chem. 2013;288(18):13022-35.

19. Masilamoni JG, Jesudason EP, Bharathi SN, Jayakumar R. The protective effect of alpha-crystallin against acute inflammation in mice. Biochim Biophys Acta. 2005;1740(3):411-20.

20. Masilamoni JG, Vignesh S, Kirubagaran R, Jesudason EP, Jayakumar R. The neuroprotective efficacy of alpha-crystallin against acute inflammation in mice. Brain Res Bull. 2005;67(3):235-41.

21. Simirskii VN, Panova IG, Sologub AA, Aleinikova KS. [Localization of crystallins in Muellerian cells in the grass frog retina]. Ontogenez. 2003;34(5):365-70.

22. Zayas-Santiago A, Rios DS, Zueva LV, Inyushin MY. Localization of alphaA-Crystallin in Rat Retinal Muller Glial Cells and Photoreceptors. Microsc Microanal. 2018;24(5):545-52.

23. Cherian M, Abraham EC. Decreased molecular chaperone property of alpha-crystallins due to posttranslational modifications. Biochem Biophys Res Commun. 1995;208(2):675-9.

24. Cherian M, Smith JB, Jiang XY, Abraham EC. Influence of protein-glutathione mixed disulfide on the chaperone-like function of alpha-crystallin. J Biol Chem. 1997;272(46):29099-103.

25. Ciano M, Allocca S, Ciardulli MC, Della Volpe L, Bonatti S, D'Agostino M. Differential phosphorylationbased regulation of alphaB-crystallin chaperone activity for multipass transmembrane proteins. Biochem Biophys Res Commun. 2016;479(2):325-30. 
26. Kamei A, Iwase H, Masuda K. Cleavage of amino acid residue(s) from the $\mathrm{N}$-terminal region of alpha A- and alpha B-crystallins in human crystalline lens during aging. Biochem Biophys Res Commun. 1997;231(2):373-8.

27. Morrison LE, Hoover HE, Thuerauf DJ, Glembotski CC. Mimicking phosphorylation of alphaBcrystallin on serine- 59 is necessary and sufficient to provide maximal protection of cardiac myocytes from apoptosis. Circ Res. 2003;92(2):203-11.

28. Maddala R, Rao VP. alpha-Crystallin localizes to the leading edges of migrating lens epithelial cells. Exp Cell Res. 2005;306(1):203-15.

29. Heise EA, Marozas LM, Grafton SA, Green KM, Kirwin SJ, Fort PE. Strain-independent increases of crystallin proteins in the retina of type 1 diabetic rats. PLoS One. 2013;8(12):e82520.

30. Kim YH, Choi MY, Kim YS, Han JM, Lee JH, Park CH, et al. Protein kinase C delta regulates antiapoptotic alphaB-crystallin in the retina of type 2 diabetes. Neurobiol Dis. 2007;28(3):293-303.

31. Rao NA, Saraswathy S, Pararajasegaram G, Bhat SP. Small heat shock protein alphaA-crystallin prevents photoreceptor degeneration in experimental autoimmune uveitis. PLoS One. 2012;7(3):e33582.

32. Sarthy VP, Brodjian SJ, Dutt K, Kennedy BN, French RP, Crabb JW. Establishment and characterization of a retinal Muller cell line. Invest Ophthalmol Vis Sci. 1998;39(1):212-6.

33. Hicks $D$, Courtois $Y$. The growth and behaviour of rat retinal Muller cells in vitro. 1. An improved method for isolation and culture. Exp Eye Res. 1990;51(2):119-29.

34. Brady JP, Garland D, Duglas-Tabor Y, Robison WG, Jr., Groome A, Wawrousek EF. Targeted disruption of the mouse alpha A-crystallin gene induces cataract and cytoplasmic inclusion bodies containing the small heat shock protein alpha B-crystallin. Proc Natl Acad Sci U S A. 1997;94(3):884-9.

35. Suzuki K, Bose P, Leong-Quong RY, Fujita DJ, Riabowol K. REAP: A two minute cell fractionation method. BMC Res Notes. 2010;3:294.

36. Sambrook J, Russell DW. Purification of nucleic acids by extraction with phenol:chloroform. CSH Protoc. 2006;2006(1).

37. Christiansen T, Richelsen B, Bruun JM. Monocyte chemoattractant protein-1 is produced in isolated adipocytes, associated with adiposity and reduced after weight loss in morbid obese subjects. Int $\mathrm{J}$ Obes (Lond). 2005;29(1):146-50.

38. Ijima R, Kaneko H, Ye F, Nagasaka Y, Takayama K, Kataoka K, et al. Interleukin-18 induces retinal pigment epithelium degeneration in mice. Invest Ophthalmol Vis Sci. 2014;55(10):6673-8.

39. Zhao M, Bai Y, Xie W, Shi X, Li F, Yang F, et al. Interleukin-1 beta Level Is Increased in Vitreous of Patients with Neovascular Age-Related Macular Degeneration (nAMD) and Polypoidal Choroidal Vasculopathy (PCV). PLoS One. 2015;10(5):e0125150.

40. Coughlin BA, Feenstra DJ, Mohr S. Muller cells and diabetic retinopathy. Vision Res. 2017;139:93100. 
41. Mohammad G, AlSharif HM, Siddiquei MM, Ahmad A, Alam K, Abu El-Asrar AM. Rho-Associated Protein Kinase-1 Mediates the Regulation of Inflammatory Markers in Diabetic Retina and in Retinal Muller Cells. Ann Clin Lab Sci. 2018;48(2):137-45.

42. Sigurdardottir S, Zapadka TE, Lindstrom SI, Liu H, Taylor BE, Lee CA, et al. Diabetes-mediated IL-17A enhances retinal inflammation, oxidative stress, and vascular permeability. Cell Immunol. 2019;341:103921.

43. Zhou T, Che D, Lan Y, Fang Z, Xie J, Gong H, et al. Mesenchymal marker expression is elevated in Muller cells exposed to high glucose and in animal models of diabetic retinopathy. Oncotarget. 2017;8(3):4582-94.

44. Li S, Yang H, Chen X. Protective effects of sulforaphane on diabetic retinopathy: activation of the Nrf2 pathway and inhibition of NLRP3 inflammasome formation. Exp Anim. 2019;68(2):221-31.

45. Yang J, Chen C, McLaughlin T, Wang Y, Le YZ, Wang JJ, et al. Loss of X-box binding protein 1 in Muller cells augments retinal inflammation in a mouse model of diabetes. Diabetologia. 2019;62(3):531-43.

46. Hoover HE, Thuerauf DJ, Martindale JJ, Glembotski CC. alpha B-crystallin gene induction and phosphorylation by MKK6-activated p38. A potential role for alpha B-crystallin as a target of the p38 branch of the cardiac stress response. J Biol Chem. 2000;275(31):23825-33.

47. Piao CS, Kim SW, Kim JB, Lee JK. Co-induction of alphaB-crystallin and MAPKAPK-2 in astrocytes in the penumbra after transient focal cerebral ischemia. Exp Brain Res. 2005;163(4):421-9.

48. Li R, Zhu Z, Reiser G. Specific phosphorylation of alphaA-crystallin is required for the alphaAcrystallin-induced protection of astrocytes against staurosporine and C2-ceramide toxicity. Neurochem Int. 2012;60(6):652-8.

49. Webster KA. Serine phosphorylation and suppression of apoptosis by the small heat shock protein alphaB-crystallin. Circ Res. 2003;92(2):130-2.

50. Potilinski MC, Lorenc V, Perisset S, Gallo JE. Mechanisms behind Retinal Ganglion Cell Loss in Diabetes and Therapeutic Approach. Int J Mol Sci. 2020;21(7).

51. Chiesa R, Gawinowicz-Kolks MA, Spector A. The phosphorylation of the primary gene products of alpha-crystallin. J Biol Chem. 1987;262(4):1438-41.

52. Kannan R, Sreekumar PG, Hinton DR. Novel roles for alpha-crystallins in retinal function and disease. Prog Retin Eye Res. 2012;31(6):576-604.

53. Voorter CE, Mulders JW, Bloemendal H, de Jong WW. Some aspects of the phosphorylation of alphacrystallin A. Eur J Biochem. 1986;160(1):203-10.

54. Harada C, Okumura A, Namekata K, Nakamura K, Mitamura Y, Ohguro H, et al. Role of monocyte chemotactic protein-1 and nuclear factor kappa $B$ in the pathogenesis of proliferative diabetic retinopathy. Diabetes Res Clin Pract. 2006;74(3):249-56.

55. Meleth AD, Agron E, Chan CC, Reed GF, Arora K, Byrnes G, et al. Serum inflammatory markers in diabetic retinopathy. Invest Ophthalmol Vis Sci. 2005;46(11):4295-301. 
56. Kumar A, Pandey RK, Miller LJ, Singh PK, Kanwar M. Muller glia in retinal innate immunity: a perspective on their roles in endophthalmitis. Crit Rev Immunol. 2013;33(2):119-35.

57. Kumar A, Shamsuddin N. Retinal Muller glia initiate innate response to infectious stimuli via toll-like receptor signaling. PLoS One. 2012;7(1):e29830.

58. Funatsu H, Noma H, Mimura T, Eguchi S, Hori S. Association of vitreous inflammatory factors with diabetic macular edema. Ophthalmology. 2009;116(1):73-9.

59. Vujosevic S, Simo R. Local and Systemic Inflammatory Biomarkers of Diabetic Retinopathy: An Integrative Approach. Invest Ophthalmol Vis Sci. 2017;58(6):BIO68-BI075.

60. Chaurasia SS, Lim RR, Parikh BH, Wey YS, Tun BB, Wong TY, et al. The NLRP3 Inflammasome May Contribute to Pathologic Neovascularization in the Advanced Stages of Diabetic Retinopathy. Sci Rep. 2018;8(1):2847.

61. Patel S, Santani D. Role of NF-kappa B in the pathogenesis of diabetes and its associated complications. Pharmacol Rep. 2009;61(4):595-603.

62. Suryavanshi SV, Kulkarni YA. NF-kappabeta: A Potential Target in the Management of Vascular Complications of Diabetes. Front Pharmacol. 2017;8:798.

\section{Table}

Due to technical limitations, Table 1 is only available as a download in the supplementary files section.

\section{Figures}


A

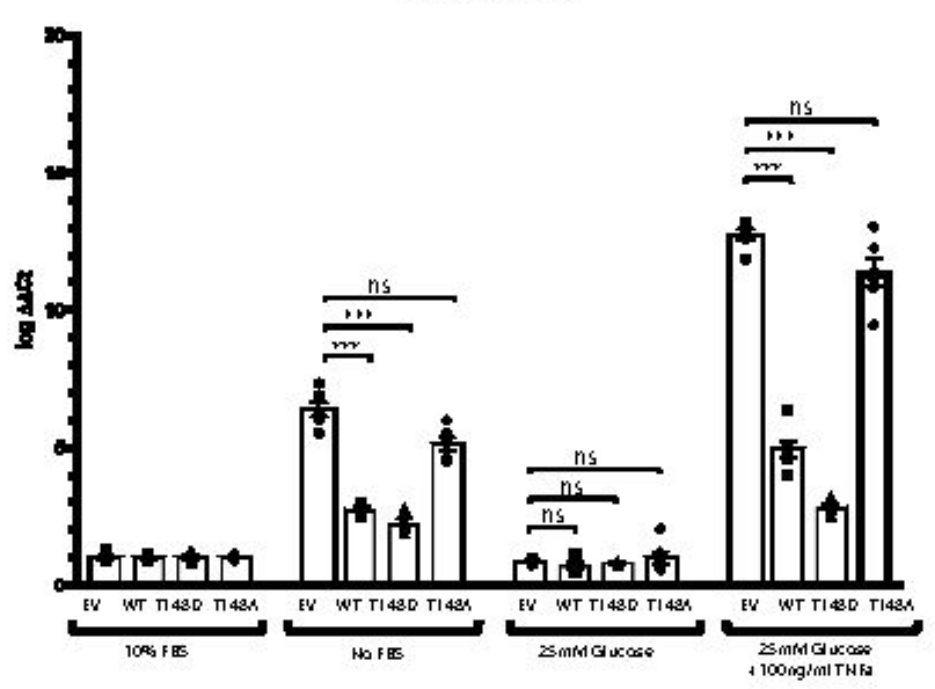

C

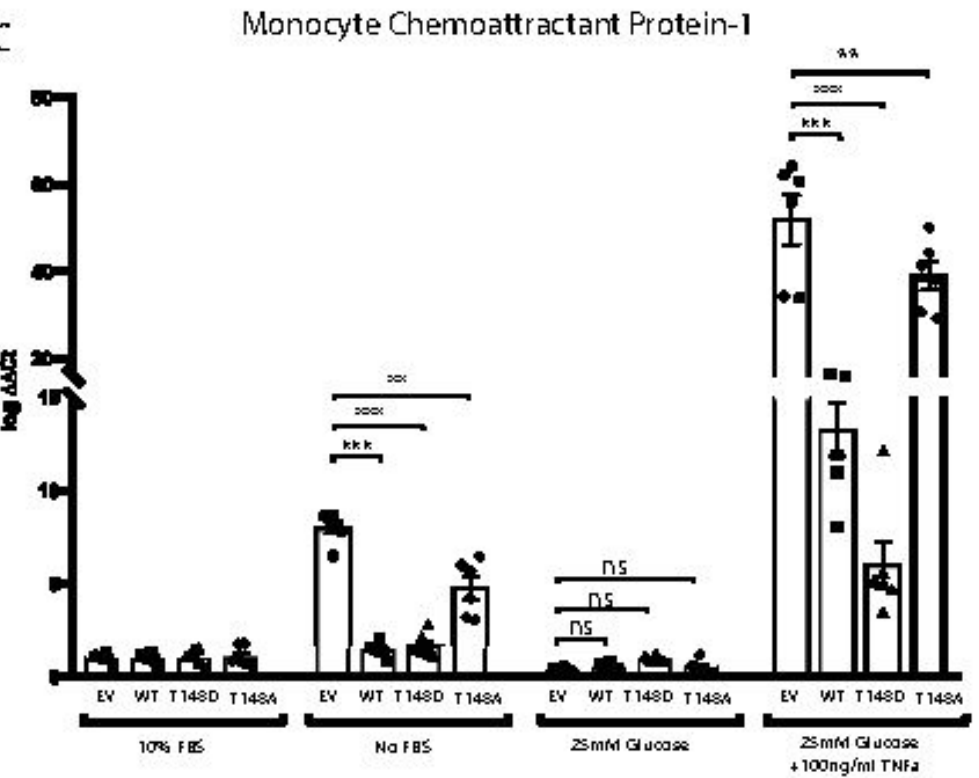

B

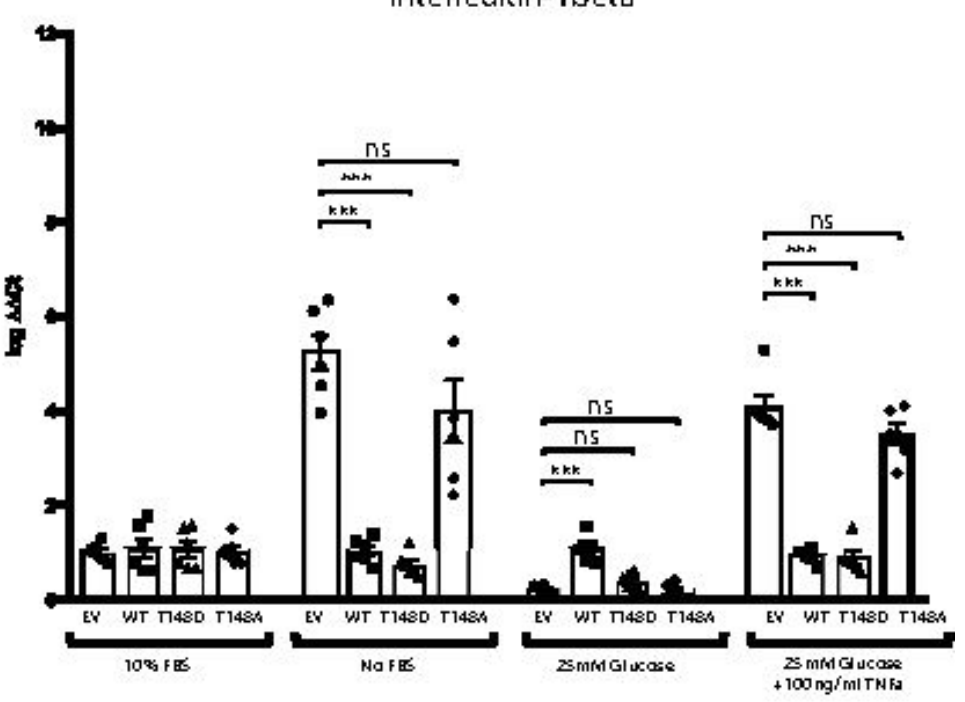

D

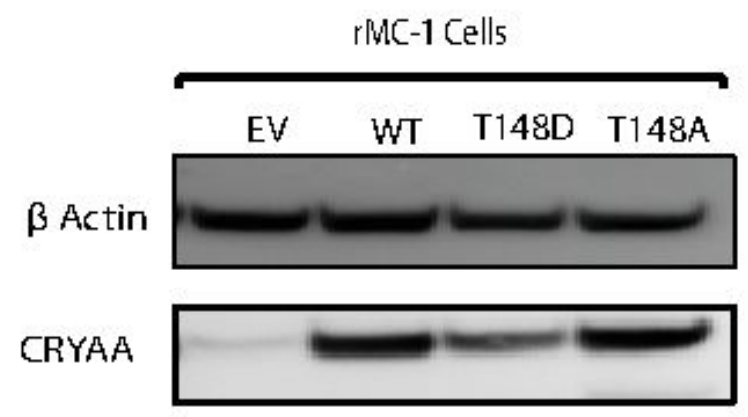

Figure 1

Metabolic stress-induced expression of pro-inflammatory mediator is regulated by alphaA-crystallin expression. rMC-1 cells were transfected with either empty vector (EV), wild type alphaA-crystallin (WT), phosphomimetic form of alphaA-crystallin (T148D) or non-phosphorylatable form of alphaA-crystallin (T148A). 24 hours post transfection, cells were either exposed to normal media (10\% FBS), serum starvation (No FBS), high glucose (25mM Glucose) or to diabetic like stress (25mM glucose $+100 \mathrm{ng} / \mathrm{ml}$ TNFa) for 4 hours. The mRNA levels of A) Interleukin-1beta, B) Interleukin-6, C) monocyte chemoattractant protein-1, were normalized to the actin-encoding gene Actb. D) representative immunoblot depicting the expression of aA-crystallin in transfected $r M C-1$ cells. (*P $\leq 0.05)$, (**P $\leq 0.01)$, $(\star \star \star P \leq 0.001)$, significantly different from respective EV-transfected cells. Each endpoint was measured 
on a minimum of 6 technical replicates. Statistical analysis was performed by 1-way ANOVA followed by Student-Newman-Keuls test.
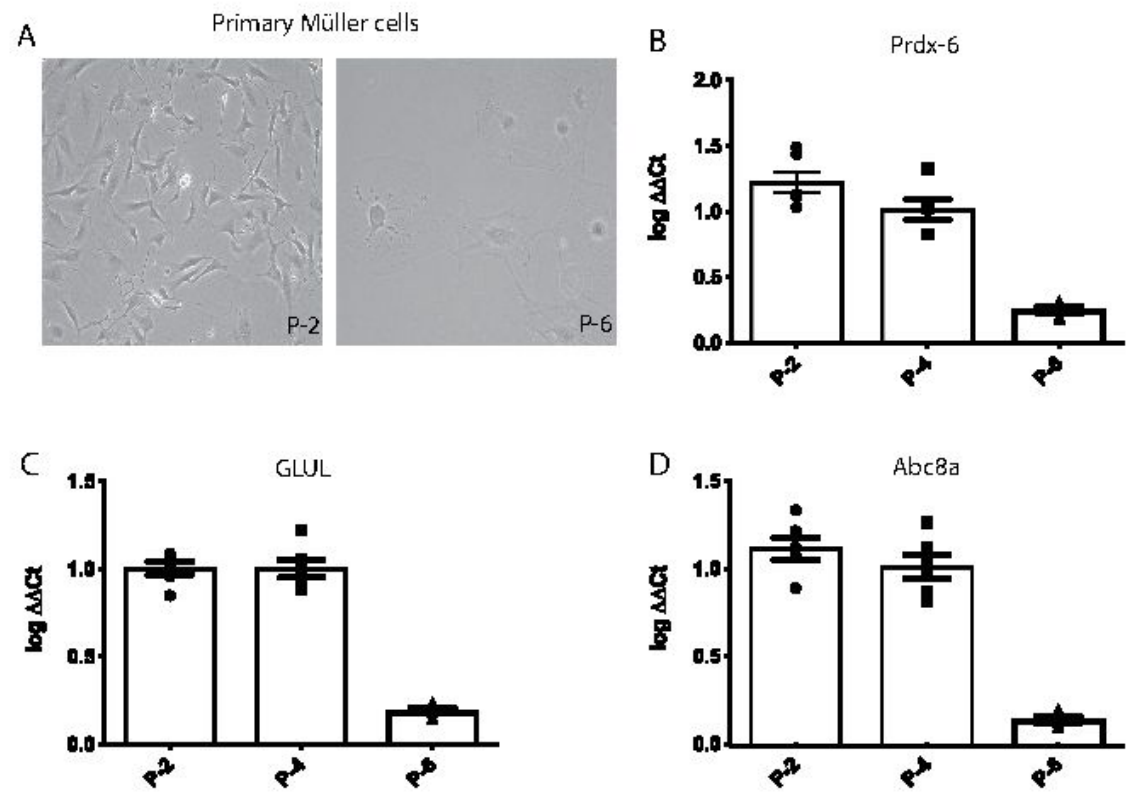

\section{Figure 2}

Primary Müller cells isolation from alphaA-crystallin knockout mice retain MGCs characteristics. Primary mouse MGCs retain an elongated morphology during the first few passages but become very flat by passage $6(A)$. The isolated Müller cells show expression of specific markers such as peroxiredoxin- 6 (Prdx-6), glutamine synthetase (GLUL) and ATP-binding cassette 8a (Abc8a). Representative graphs of mRNA levels of B) Prdx-6, C) GLUL and D) Abc8a in primary Müller cells at passage-2 (P-2), Passage-4 (P4) and Passage-6 (P-6) are shown normalized to the actin-encoding gene Actb. The Müller cell specific markers are highly expressed until they dramatically decrease after passage 6 . 
A

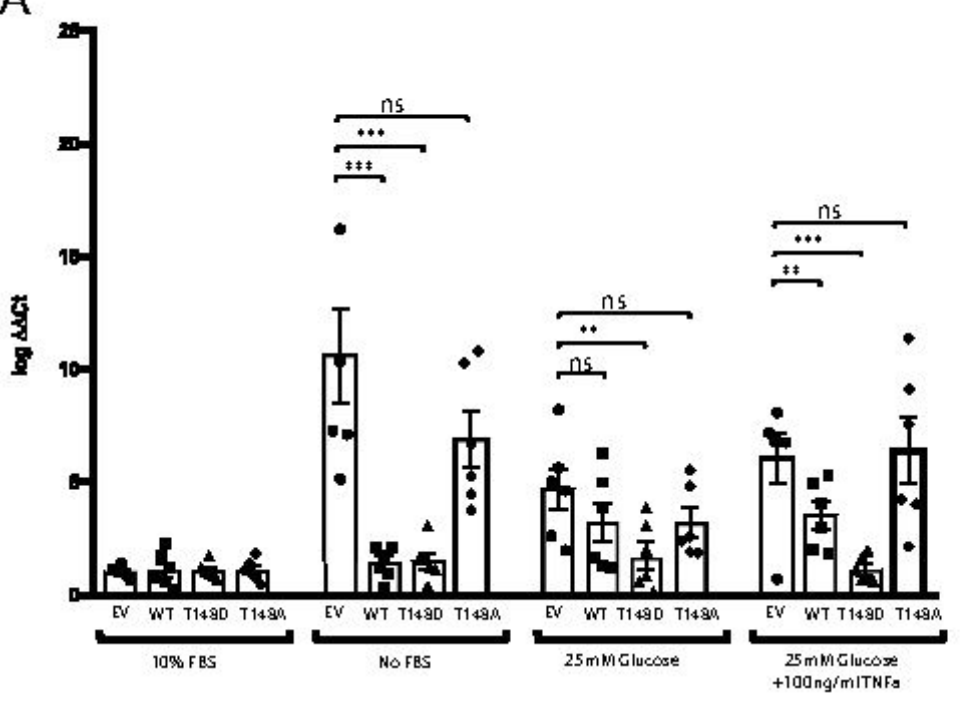

Interleukin-18

$c$

C.

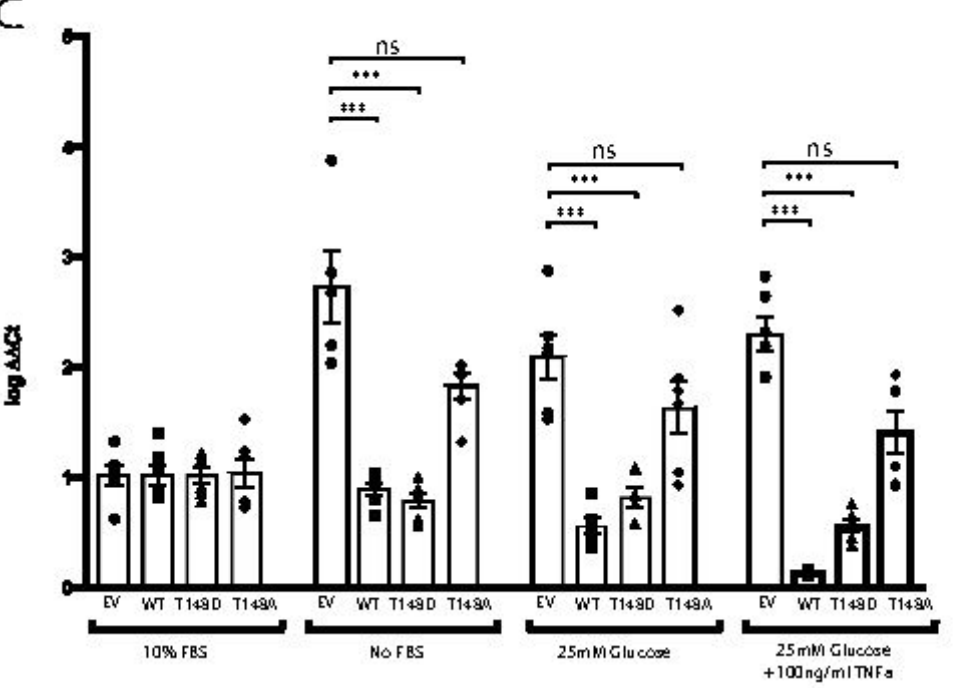

$E$

AKO-MGC5
B

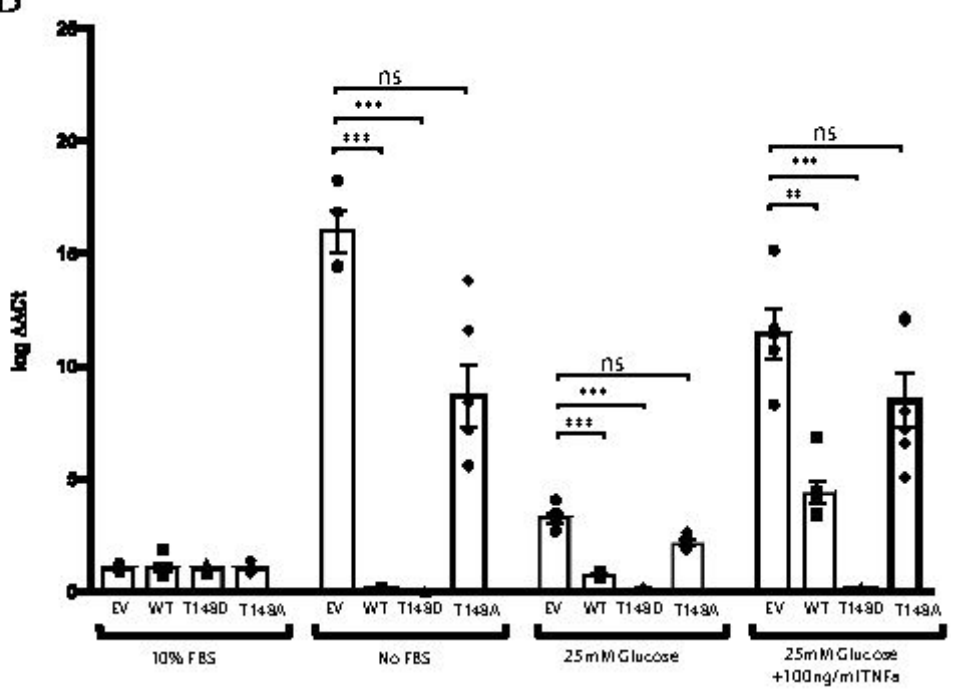

Monocyte Chemoattractant Protein-1

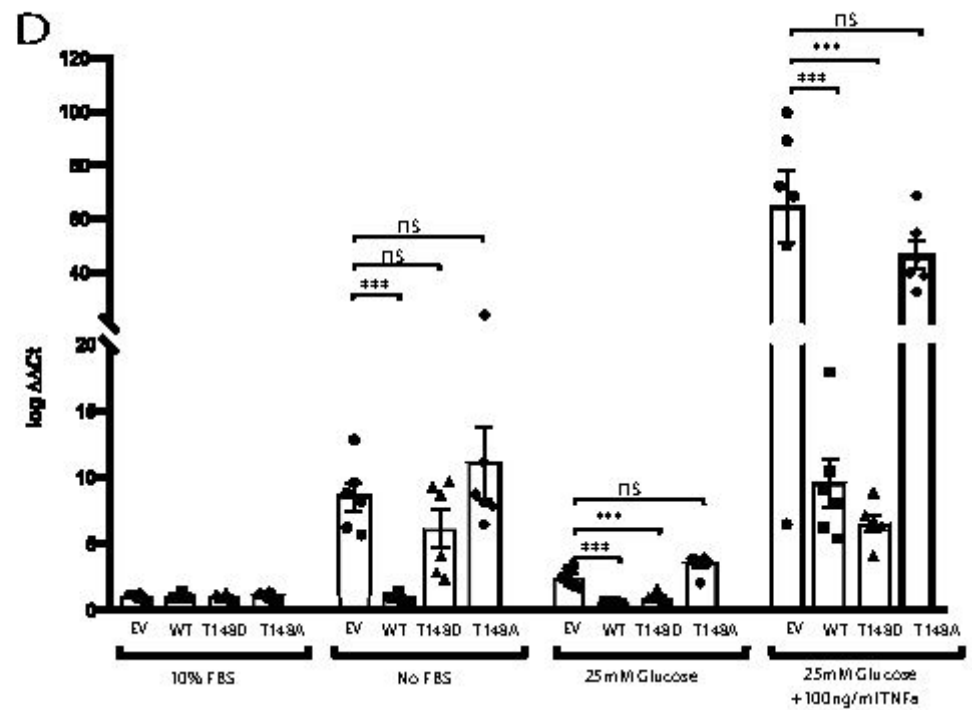

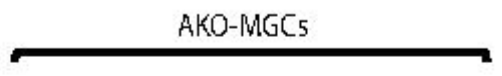

EV WT T148D T148A

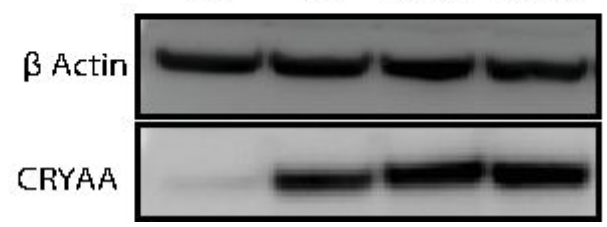

Figure 3

Primary MGCs express pro-inflammatory molecules upon stress exposure, which is modulated by aAcrystallin. Primary Müller cells were transfected with either empty vector (EV), alphaA-wild type crystallin (WT), phosphomimetic form of alphaA-crystallin (T148D) or non-phosphorylatable form of alphaAcrystallin (T148A). 24 hours post transfection, cells were either exposed to normal media (10\% FBS), serum starvation (No FBS), high glucose ( $25 \mathrm{mM}$ Glucose) or to diabetic like stress (25mM glucose $+100 \mathrm{ng} / \mathrm{ml} \mathrm{TNFa}$ ) for 4 hours. The mRNA levels of A) Interleukin-1beta, B) Interleukin-6, C) Interleukin-18 
and D) monocyte chemoattractant protein-1, were normalized to the actin-encoding gene Actb. E) Representative immunoblot depicting the expression of aA-crystallin in transfected MGCs. ( $\left.{ }^{\star} P \leq 0.05\right)$, ( $\left.{ }^{\star * P} \leq 0.01\right)$, ( $\left.{ }^{\star \star *} P \leq 0.001\right)$, significantly different from respective EV-transfected cells. The primary Müller cells were used upto passage- 5 for the experiments. Each endpoint was measured on a minimum of 6 technical replicates in 3 independent experiments. Statistical analysis was performed by 1-way ANOVA followed by Student-Newman-Keuls test.
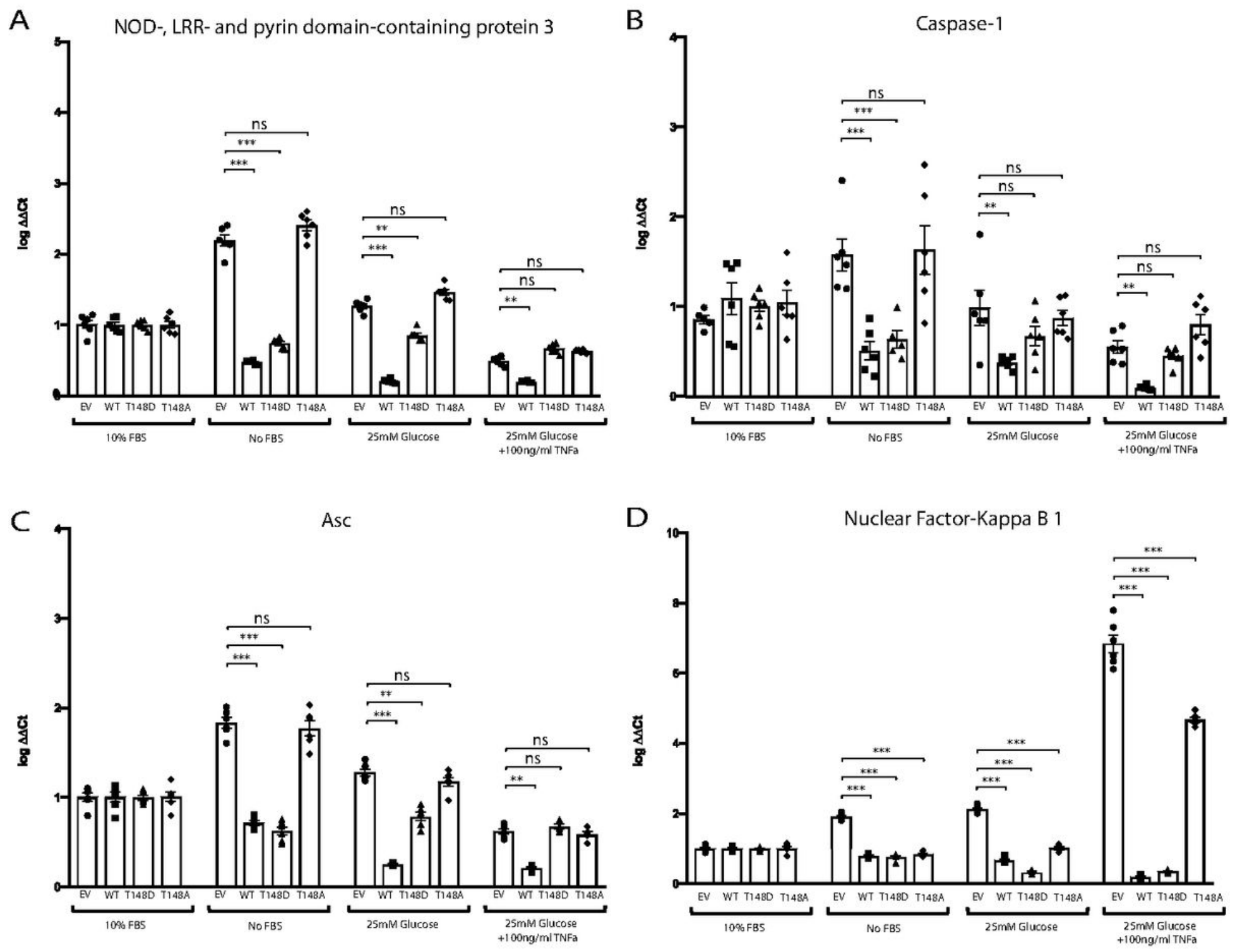

Figure 4

aA-crystallin distinct interaction with inflammatory pathways might play key role in regulating neuroinflammation in MGCs. Primary Müller cells were transfected with either empty vector (EV), alphaA-wild type crystallin (WT), phosphomimetic form of alphaA-crystallin (T148D) or non-phosphorylatable form of alphaA-crystallin (T148A). 24 hours post transfection, cells were either exposed to normal media (10\% 
FBS), serum starvation (No FBS), high glucose ( $25 \mathrm{mM}$ Glucose) or to diabetic like stress (25mM glucose $+100 \mathrm{ng} / \mathrm{ml} \mathrm{TNFa}$ ) for 4 hours. The mRNA levels of A) NOD-, LRR- and pyrin domain-containing protein 3 (Nlrp3) B) Caspase-1 (Casp-1), C) Apoptosis-associated speck like protein containing a caspase recruitment domain (Asc) and D) Nuclear Factor-Kappa B-1 (Nf-kB1), were normalized to the actinencoding gene Actb. ( $\left.{ }^{\star} P \leq 0.05\right)$, ( $\left.{ }^{\star} P \leq \leq 0.01\right)$, ( $\left.{ }^{\star \star \star} P \leq 0.001\right)$, significantly different from respective EVtransfected cells. The primary Müller cells were used upto passage- 5 for the experiments. Each endpoint was measured on a minimum of 6 technical replicates. Statistical analysis was performed by 1-way ANOVA followed by Student-Newman-Keuls test. 
A

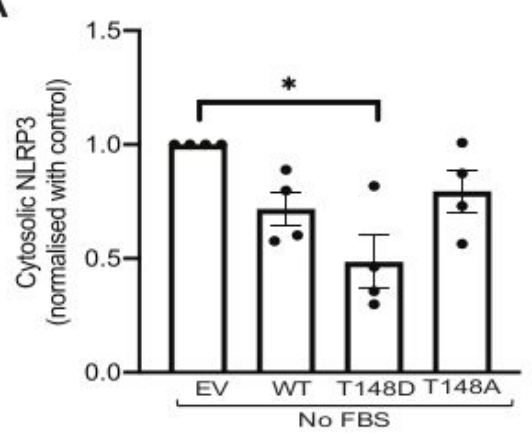

C

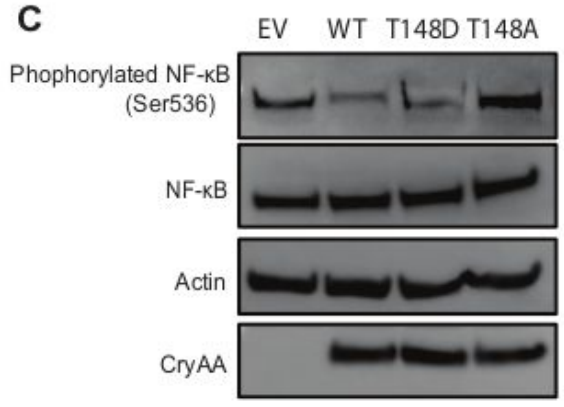

E

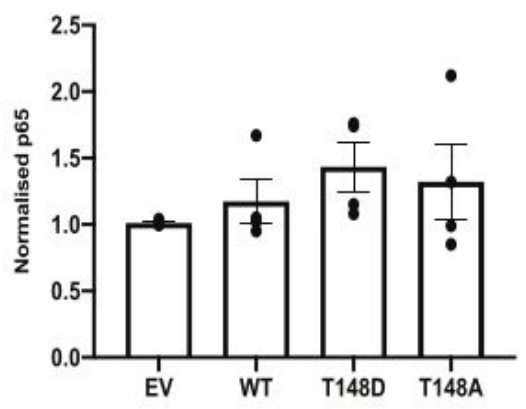

B
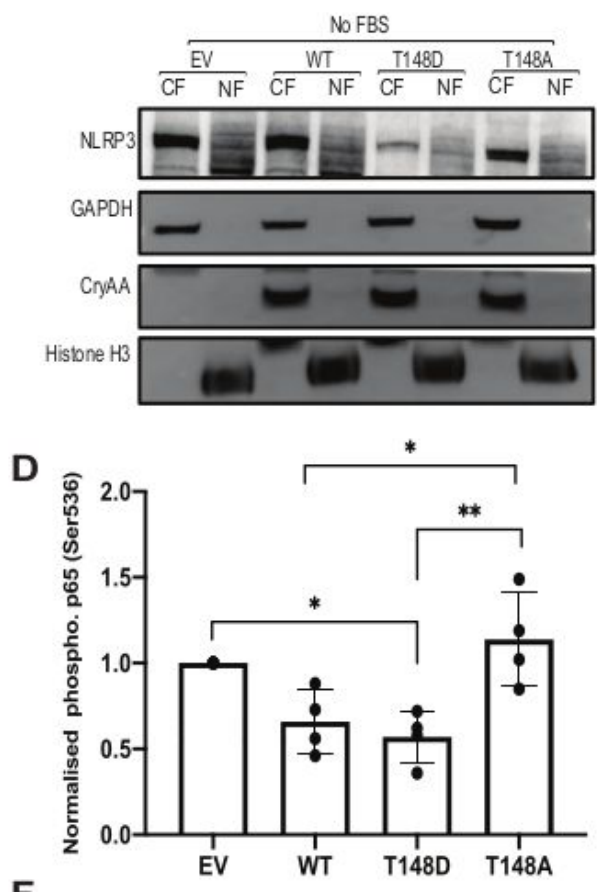

F

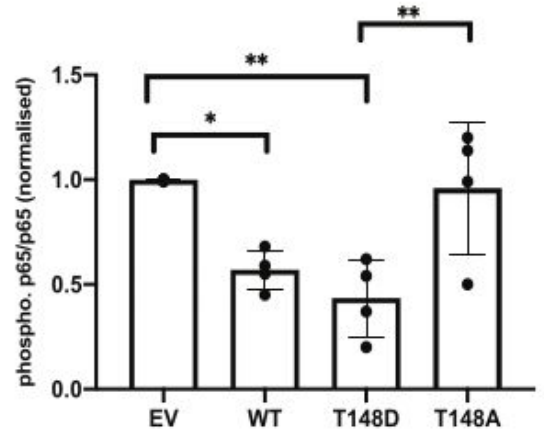

\section{Figure 5}

aA-crystallin regulates neuro-inflammation in MGCs through inhibiting NLRP3 induction and NF-kB activation. Primary Müller cells were transfected with either empty vector (EV), alphaA-wild type crystallin (WT), phosphomimetic form of alphaA-crystallin (T148D) or non-phosphorylatable form of alphaAcrystallin (T148A). 24 hours post transfection, cells were either exposed to diabetic like stress (25mM glucose +100ng/ml TNFa) for 4 hours. A) The expression of NLRP3 was assessed in cytosolic fractions 
were normalized to the empty vector. B) The cytosolic fraction was blotted for anti-NLRP3, anti- aAcrystallin where GAPDH and Histone-H3 served as the cytosolic (CF) and nuclear fractions (NF) controls respectively. C) The total lysate was blotted for anti-phosphorylated NF-kB (Ser536), anti-NF-kB, anti- aAcrystallin and anti-actin was used as loading control. The fold change in the protein levels of $D$ ) phosphorylated NF-kB (Ser536) and ratio of phosphorylated NF-kB (Ser536) and total NF-kB was analyzed were normalized to the empty vector. ( $\left.{ }^{\star} P \leq 0.05\right)$, ( $\left.{ }^{\star \star} P \leq 0.01\right)$, ( $\left.{ }^{\star \star *} P \leq 0.001\right)$, significantly different from respective EV-transfected cells. The primary Müller cells were used upto passage- 5 for the experiments. Each endpoint was measured on a minimum of 3 technical replicates. Statistical analysis was performed by 1-way ANOVA followed by Student-Newman-Keuls test.

\section{Supplementary Files}

This is a list of supplementary files associated with this preprint. Click to download.

- Table1Genesequence.pdf 